\title{
Prevalence of hypertension in overweight and obese children from a large school-based population in Shanghai, China
}

\author{
Xi Lu ${ }^{1 *}$, Peng Shi ${ }^{2}$, Chun-Yan Luo ${ }^{1}$, Yue-Fang Zhou ${ }^{1}$, Hui-Ting Yu' ${ }^{1}$, Chang-Yi Guo and Fan Wu ${ }^{1 *}$
}

\begin{abstract}
Background: The ongoing rise in the prevalence of hypertension in children and adolescents is considered to be accompanied with the epidemic of childhood overweight and obesity. In this study, we established a large scale cross-sectional study in Shanghai, China, which presented a new evidence for the correlation of hypertension prevalence with overweight and obesity stages in Chinese children and adolescents.

Methods: A school-based cross-sectional study was conducted during February to December 2009 in Shanghai, China, including total 78,114 children and adolescents. Body weight, height, waist circumference (WC) and blood pressure (BP) were measured. Overweight and obesity were defined according to sex- and age- specific Chinese reference data.
\end{abstract}

Results: Both SBP and DBP were very significantly increased in overweight (OW) and obese (OB) groups. With age and sex controlled, BMI and WC were independently positively correlated with SBP and DBP. The prevalence of high SBP, DBP and hypertension were markedly higher among OW and OB children than normal weight (NW) group. Odds ratios (ORs) for high SBP, high DBP and high BP were significantly greater in OW and OB children than NW group, and showed a trend increase correlating with obesity stages (all $P<0.0001$ ). According to the increasing OR with different combination of obese status of BMI and WC, WC has a stronger influence on hypertension. The combination of BMI and WC obese shows substantially higher ORs compared with those for either BMI or WC obese alone.

Conclusions: In this study on a large school-based population in Shanghai, China, BMI and WC are positively correlated with SBP and DBP. Being overweight or obese greatly increased the risk of hypertension in Chinese children and adolescents, in which WC is considered as a more sensitive indicator than BMI.

Keywords: Obesity, Overweight, Hypertension, Children and adolescents, China

\section{Background}

From the recent announcement of National CDC of China, hypertension has become a major public health problem in China. In the 2010 Chinese guidelines for the management of hypertension, the prevalence of hypertension has been increasing in China for decades, and reached $18.8 \%$ in the year 2002 [1]. Importantly, these adulthood hypertension can be originally observed to extend back to the childhood. More and more

\footnotetext{
* Correspondence: lucy-luxi@163.com; Fwu@scdc.sh.cn

'Shanghai Municipal Center for Disease Control and Prevention \& Shanghai Institutes of Preventive Medicine, 1380 W. Zhongshan Road, Shanghai 200336, China

Full list of author information is available at the end of the article
}

evidence showed hypertensive children are more likely to develop hypertension in adulthood [2-4]. The increase of childhood hypertension not only increases the prevalence of hypertension in later adulthood decades, but also further correlates with increased cardiovascular morbidituy and mortality [5]. Therefore, the study on the prevalence and early diagnosis of hypertension in childhood is an important strategy for the public control and prevention of cardiovascular diseases.

Although the childhood hypertension was once considered to be rare in pediatrics, the ongoing rise in the prevalence of hypertension becomes a common problem in children and adolescents, which is considered to be

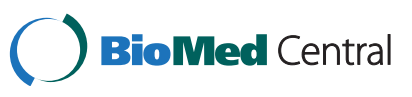


accompanied with the epidemic of childhood overweight and obesity [6]. Several studies showed that obesity is a well predictor of hypertension [7-9]. However, this correlation of hypertension with obesity in childhood lacks the confirmation from large scale cohort of children and adolescents in China.

Shanghai is the largest city in China, with about 2 million ongoing school children population. Importantly, among them the obese prevalence has greatly increased during the past 20 years [10]. From the statistic data in Shanghai CDC, the obesity prevalence in ongoing children of Shanghai was $3.76 \%$ in 1991, but in 2009 , the obesity prevalence was increased to be $13.53 \%$, which means now there are more than 200 thousand obese children in Shanghai. Meanwhile, the overweight prevalence was $11.27 \%$. How to take care of these children's health, especially monitoring the accompanied risk factors, has become an important issue. Therefore, our center established a large scale crosssectional study, named "Shanghai children and adolescents disease risk factor study" (SCADRFS), in Shanghai from 2009, in which 78,114 children including 40,105 boys and 38,009 girls were observed on their obesity and hypertension stages. Our study present a new evidence for the correlation of hypertension prevalence with overweight and obesity stages in a large population of Chinese children and adolescents.

\section{Methods}

\section{Study subjects}

A school-based cross-sectional study was conducted during the period February 2009 to December 2009 in Shanghai, China. Two-stage cluster sampling technique was used to choose the study sample. A total sample of 78,114 children and adolescents including 40,105 boys and 38,009 girls from the selected schools constituted the subjects of the study. Written informed consent form was obtained from adolescents and their parents. This study was approved by The Ethical Committee of Shanghai CDC.

\section{Anthropometric measurements}

Body weight was determined to the nearest $0.1 \mathrm{~kg}$ on standard physician's beam scales with the children and adolescents wearing only the underwear and no shoes. Height was measured to the nearest $0.1 \mathrm{~cm}$ on standardized, wall-mounted height boards according to the following protocol: no shoes, heels together, and student's heels, buttocks, shoulders, and head touching the vertical wall surface with line of sight aligned horizontally. Waist circumference (WC) was also measured. Each of the standard physician's beam scales, wall-mounted height boards and body tape measure used to measure were calibrated previously. Body mass index (BMI) was computed by dividing weight $(\mathrm{kg})$ by height squared $\left(\mathrm{m}^{2}\right)$. The age- and gender-specific BMI and WC cutoffs newly developed by the working group on obesity in China (WGOC) were used to define overweight and obesity $[11,12]$. Overweight is defined by a BMI or WC at or above the 85 th percentile and lower than the 95th percentile for children and adolescents of the same age and sex. And obesity is defined by BMI or WC greater than the 95 th percentile.

\section{Blood pressure measurement and high blood pressure definition}

Blood pressure was measured by two trained physicians using a standard mercury sphygmomanometer at the right arm with students in the seated position after at least $5 \mathrm{~min}$ of rest. The cuff size was based on the length and circumference of the upper arm and was chosen to be as large as possible without having the elbow skin crease obstruct the stethoscope [13]. Blood pressure values were approximated to the nearest $2 \mathrm{mmHg}$. Systolic blood pressure (SBP) was defined by the first Korotkoff sound (appearance of sounds), and diastolic blood pressure (DBP) was defined by the fourth Korotkoff sound (sound muffling). In order to make the children comfortable in a relaxed environment, measurements were taken in the classroom in the presence of their classmates and teachers without specifying that doctors were performing the activity and doctors wore casual clothes. The age- and genderspecific blood pressure cutoffs in Chinese children and adolescents were used to define pre-hypertension and hypertension [14]. In this definition, hypertension was defined as SBP and/or DBP above the 95th percentile for age and gender.

\section{Statistical analysis}

The continuous variables, including age, weight, height, BMI, and blood pressure, were summarized by the mean and range. Other categorical variables were summarized by count and percentage. Logistical regression model was used to estimate the odds ratios (ORs) and 95\% confidence intervals (CIs) between weight status and blood pressure after adjustment for age and gender. Chi-square test was performed to compare the difference between OW and NW, OB and NW. Statistical tests were set with a significance level of 0.05. All data analyses were conducted by using SPSS 15.0 (SPSS Inc., Chicago, Illinois, USA).

\section{Results}

To make an overall enrollment in Shanghai, we studied a large scale of children population covering 110 schools from every district in the city including 60 schools in 9 urban districts and 50 schools in 10 rural 
Table 1 Baseline characteristics of the study population stratified by BMI and WC

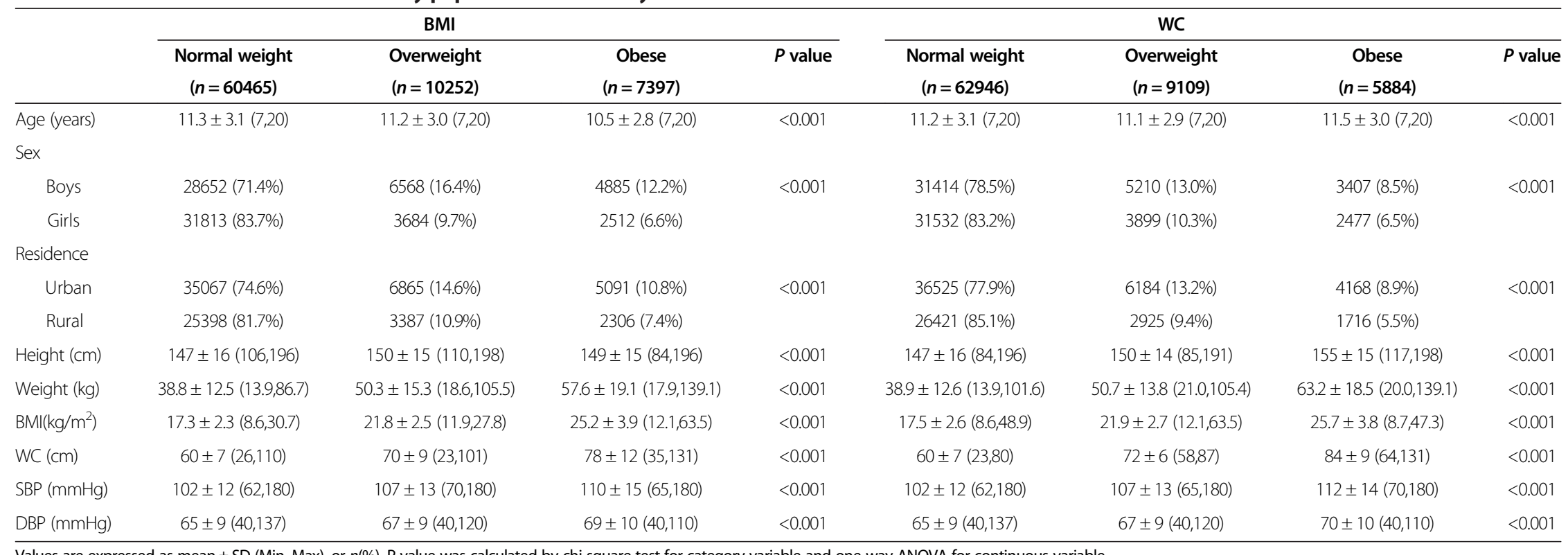

Values are expressed as mean + SD (Min, Max), or $n(\%)$. P value was calculated by chi-square test for category variable and one-way ANOVA for continuous variable. BMI, body mass index; WC, waist circumference; DBP, diastolic blood pressure; SBP, systolic blood pressure. 
Table 2 Estimated odds ratios and $95 \%$ confidence intervals for normal weight, overweight and obese classified by BMI and WC after adjusting for age and sex

\begin{tabular}{|c|c|c|c|c|c|c|c|c|c|c|c|c|c|}
\hline & & \multicolumn{4}{|c|}{ High SBP vs normal SBP } & \multicolumn{4}{|c|}{ High DBP vs normal DBP } & \multicolumn{4}{|c|}{ High BP vs normal BP } \\
\hline & & $n(\%)$ & OR & $95 \% \mathrm{Cl}$ & $P$ & $n(\%)$ & OR & $95 \% \mathrm{Cl}$ & $P$ & $n(\%)$ & OR & $95 \% \mathrm{Cl}$ & $P$ \\
\hline \multicolumn{14}{|l|}{ BMI } \\
\hline & NW & $3808(6.30)$ & 1.00 & & & $2689(4.45)$ & 1.00 & & & $5243(8.67)$ & 1.00 & & \\
\hline & OW & $1362(13.29)$ & 1.67 & $1.54-1.82$ & $<0.0001$ & $789(7.70)$ & 1.51 & $1.36-1.67$ & $<0.0001$ & $1630(15.90)$ & 1.50 & $1.39-1.62$ & $<0.0001$ \\
\hline & $\mathrm{OB}$ & $1600(21.63)$ & 2.17 & $1.94-2.43$ & $<0.0001$ & $992(13.41)$ & 2.09 & $1.83-2.39$ & $<0.0001$ & $1896(25.63)$ & 1.95 & $1.76-2.16$ & $<0.0001$ \\
\hline \multicolumn{14}{|l|}{ WC } \\
\hline & NW & $4110(6.53)$ & 1.00 & & & $2894(4.60)$ & 1.00 & & & $5577(8.86)$ & 1.00 & & \\
\hline & OW & $1261(13.84)$ & 1.48 & $1.35-1.62$ & $<0.0001$ & $784(8.61)$ & 1.35 & $1.21-1.50$ & $<0.0001$ & $1547(16.98)$ & 1.47 & $1.36-1.60$ & $<0.0001$ \\
\hline & $\mathrm{OB}$ & $1386(23.56)$ & 2.25 & $2.01-2.53$ & $<0.0001$ & 785 (13.34) & 1.79 & $1.56-2.06$ & $<0.0001$ & $1629(27.69)$ & 2.24 & $2.02-2.48$ & $<0.0001$ \\
\hline Age & & & 1.03 & $1.02-1.04$ & $<0.0001$ & & 0.93 & $0.92-0.94$ & $<0.0001$ & & 1.01 & $1.00-1.02$ & 0.0255 \\
\hline \multicolumn{14}{|l|}{ Sex } \\
\hline & Girls vs boys & & 0.78 & $0.74-0.82$ & $<0.0001$ & & 1.14 & $1.07-1.21$ & $<0.0001$ & & 0.90 & $0.86-0.94$ & $<0.0001$ \\
\hline
\end{tabular}

DBP, diastolic blood pressure; SBP, systolic blood pressure; BMI, body mass index; WC, waist circumference; NW, normal weight; OW, overweight; OB, obese. 
districts. As shown in Table 1, totally 78,114 children and adolescents were enrolled for the medical examination, among which 175 cases missing waist circumference data. The average age of the students was around 11 years old (range 7-20 years). Boys in both BMI and WC overweight/obese groups are significantly more than girls (BMI group: OW 16.4\% vs.9.7\%, OB 12.2\% vs.6.6\%; WC group: OW $13.0 \%$ vs.10.3\%, OB $8.5 \%$ vs.6.5\%). There are more children in urban districts with overweight/obese status than those in rural districts (BMI group: OW 14.6\% vs.10.9\%, OB 10.8\% vs.7.4\%; WC group: OW 13.2\% vs.9.4\%, OB $8.9 \%$ vs.5.5\%). As shown in Table 1, among groups stratified by $\mathrm{BMI}$ and $\mathrm{WC}$ for the main anthropometric values, both SBP and DBP were very significantly increased in OW and OB groups, which consistent with previous report that obese children have much higher blood pressure than the normal ones.

Table 2 made a further analysis on the hypertension prevalence. Classified by BMI or WC, the prevalence of high SBP increased more greatly compared with that of DBP. The prevalence of high SBP, DBP and hypertension were all markedly higher among OW and $\mathrm{OB}$ children than NW group. The risk of high BP prevalence was 1.5-2.2 folds in OW and OB children than that in NW group. Odds ratios (ORs) for high SBP, DBP and BP were significantly greater in $\mathrm{OW}$ and $\mathrm{OB}$ children than NW group, and showed a trend increase correlating with obesity stages(all P <0.0001). BMI and WC were statistically significant after adjusting age and sex.

Table 3 shows the interaction between different obese status of BMI and WC on the prevalence of high SBP, DBP and $\mathrm{BP}$ by the adjusted odds ratio (ORs) with 95\% confidence intervals (95\% CIs). Controlling for BMI, the adjusted ORs progressively increased with the increasing obese status classified by $\mathrm{WC}$, and vise visa. According to the increasing OR with different combination of obese status of BMI and $\mathrm{WC}$, we can find $\mathrm{WC}$ has a stronger influence on high SBP, high DBP and hypertension respectively. For example, when compared with students who were WC overweight, the OR of high BP became higher (1.91 for BMI NW vs. 2.64 for BMI OB, increase 38.2\%), while when compared with those who were BMI overweight, the OR of high BP became greater higher with WC obese statues increasing (1.76 for WC NW vs. 2.66 for $\mathrm{WC} \mathrm{OB}$, increase 51.1\%). Furthermore, the combination of BMI and WC obese shows substantially higher ORs compared with those for either BMI or WC obese alone, 4.66 for BMI OB* WC OB.

\section{Discussion}

Here we present a cross-sectional, school-based population study covering 110 schools from 19 districts of Shanghai with a large scale of children sample size. We found a significant correlation of children obesity with hypertension prevalence. Our study determined that $22.6 \%$ of children were in overweight or obese stage defined by BMI (19.2\% defined by WC). Among them, there were significant increase on the prevalence of high SBP, DBP and hypertension. Importantly, the logistic regression analysis showed a positively strong association of obesity stages with the risk of hypertension prevalence in these children.

Being overweight or obese has become highly prevalent in Western countries and is rapidly reaching epidemic proportions in the developing countries. Obesity-related disorders, such as hypertension and diabetes, are also increasing at an alarming rate. In the study from Robert Whitaker et al., obese children under three years of age without obese parents are at low risk for obesity in adulthood, but among older children, obesity is an increasingly important predictor of adult obesity, regardless of whether the parents are obese or not [15].

Table 3 Relationship between different categories of BMI, WC and hypertension after adjusting for age and sex

\begin{tabular}{|c|c|c|c|c|c|c|c|c|c|c|c|}
\hline \multicolumn{2}{|c|}{ Categories } & \multirow[b]{2}{*}{$N$} & \multicolumn{3}{|c|}{ High SBP vs normal SBP } & \multicolumn{3}{|c|}{ High DBP vs normal DBP } & \multicolumn{3}{|c|}{ High BP vs normal BP } \\
\hline BMI & WC & & $n(\%)$ & OR(95\% Cl) & $P$ & $n(\%)$ & OR(95\% Cl) & $P$ & $n(\%)$ & OR(95\% Cl) & $P$ \\
\hline$\overline{N W}$ & NW & 57867 & $3521(6.08)$ & 1.00 & & $2509(4.34)$ & 1.00 & & $4864(8.41)$ & 1.00 & \\
\hline NW & OW & 2211 & $248(11.22)$ & $2.00(1.75,2.30)$ & $<0.0001$ & $155(7.01)$ & $1.71(1.44,2.02)$ & $<0.0001$ & $326(14.74)$ & $1.91(1.69,2.16)$ & $<0.0001$ \\
\hline NW & $\mathrm{OB}$ & 242 & $33(13.64)$ & $2.49(1.72,3.61)$ & $<0.0001$ & $19(7.85)$ & $1.98(1.23,3.17)$ & $<0.0001$ & $42(17.36)$ & $2.32(1.66,3.24)$ & $<0.0001$ \\
\hline OW & NW & 4484 & $524(11.69)$ & $1.99(1.80,2.19)$ & $<0.0001$ & $335(7.47)$ & $1.74(1.54,1.96)$ & $<0.0001$ & $632(14.09)$ & $1.76(1.61,1.92)$ & $<0.0001$ \\
\hline ow & OW & 4621 & $645(13.96)$ & $2.41(2.20,2.63)$ & $<0.0001$ & $363(7.86)$ & $1.95(1.74,2.19)$ & $<0.0001$ & $773(16.73)$ & $2.15(1.98,2.34)$ & $<0.0001$ \\
\hline OW & $\mathrm{OB}$ & 1131 & $190(16.80)$ & $2.99(2.55,3.52)$ & $<0.0001$ & $91(8.05)$ & $2.18(1.75,2.72)$ & 0.0046 & $224(19.81)$ & $2.66(2.29,3.09)$ & $<0.0001$ \\
\hline $\mathrm{OB}$ & NW & 595 & $65(10.92)$ & $1.93(1.49,2.51)$ & $<0.0001$ & $50(8.40)$ & $1.82(1.36,2.44)$ & $<0.0001$ & 81 (13.61) & $1.72(1.36,2.18)$ & $<0.0001$ \\
\hline OB & OW & 2277 & 368 (16.16) & $2.96(2.63,3.33)$ & $<0.0001$ & $266(11.68)$ & $2.72(2.37,3.11)$ & $<0.0001$ & 448 (19.68) & $2.64(2.37,2.95)$ & $<0.0001$ \\
\hline $\mathrm{OB}$ & $\mathrm{OB}$ & 4511 & $1163(25.78)$ & $5.22(4.84,5.63)$ & $<0.0001$ & $675(14.96)$ & $3.92(3.58,4.30)$ & $<0.0001$ & $1363(30.22$ & $4.66(4.34,5.00)$ & $<0.0001$ \\
\hline
\end{tabular}

$N$ is the total number in the category. $n$ is the number in the category, and\% is the proportion in the category with outcome. Adjusted ORs with $95 \%$ confidence intervals ( $95 \% \mathrm{Cls}$ ) were computed after adjusting for age and sex. 
The criteria for definition of children's hypertension is arbitrary and to a certain extent, artificial. The normative data for BP in children and adolescents is the United States-based 2004 Task Force Report Update, which has been widely used in recent US-based or European-based studies [16]. Another widely used normative data is the report of WHO expert committee on the Hypertension Control [17]. But whether these standards are applicable to the children and adolescents in the other parts of the world, or the different ethnic backgrounds is unknown, since several studies already showed the difference of children's BP between children in China and those in western countries [18]. Therefore, in this study, we used Chinese reference standard for children's hypertension published in 2010, which was established from eleven large scale cross-sectional BP surveys in mainland China from 2001 to 2010, covering four municipalities and seven provinces. Based on the Chinese reference, we made a more suitable evaluation on the Chinese children population and classified the children with hypertension.

Compared with the widely studied on the relation of obesity with hypertension, fewer studies were investigated the prevalence of hypertension in Chinese Children and whether it is related with children's obesity in China. One recent study conducted Changsha city in China, showed the prevalence of hypertension among adolescents at the age of 12-17 years and found the relation with BMI [19]. Another study also reported the correlation of hypertension with BMI in children population in Hainan province of South China [20]. In this study, we classified the obesity stages of children both by BMI and WC, the two most widely used index for obesity. BMI is most widely used for the indicator of body fatness, but WC indicated the central fat that more likely correlates with diabetes than the association with general fat. Our results showed the overweight and obese groups have apparently higher prevalence of high DBP, high SBP and hypertension, which the obesity stages can be classified both by BMI and $\mathrm{WC}$ independently. Interestingly, our data also indicated that WC has a stronger influence on the hypertension, although one group reported that BMI is more sensitive indicator for the prevalence of hypertension in Beijing [21]. Compared with general fat defined by BMI, central obesity defined by WC is the kind of excessive abdominal fat around the stomach and abdomen, which is believed to have a strong association with several health risks, such as type 2 diabetes, asthma and cardiovascular diseases. Especially, it is reported that a central distribution of body fat is associated with increased BP, independently of body mass index and insulin resistance in the middle-aged men [22]. In our study, our finding indicated that in the childhood, the central obesity also has much more stronger association with risk of hypertension compared with general obesity.

\section{Conclusion}

In a conclusion, our large scale school-based study on children population in Shanghai showed the higher prevalence of hypertension was associated with higher BMI or WC overweight and obese children. Being overweight or obese significantly increases the risk of hypertension in the children at the age from 7 to 20 years. Furthermore, the WC seems to be a more sensitive risk factor associated with the risk of hypertension than BMI.

\section{Competing interest}

The author declares to have no proprietary, financial, professional or other interest of any nature or kind in any product, service, and/or company that could be construed as influencing the position presented in this manuscript.

\section{Authors' contributions}

$\mathrm{XL}$ and FW designed the study plan; FW, CYG, CYL, XL, and YFZ organized the school-based population, collected each data; and XL, PS, HTY and FW analyzed the data and wrote the manuscript. All authors read and approved the final manuscript.

\section{Funding}

This work was supported by the National Natural Science Foundation of China (Grant No. 81001250).

\section{Author details}

${ }^{1}$ Shanghai Municipal Center for Disease Control and Prevention \& Shanghai Institutes of Preventive Medicine, 1380 W. Zhongshan Road, Shanghai 200336, China. ²Department of Clinical Epidemiology, Children's Hospital of Fudan University, Shanghai 201102, China.

Received: 6 August 2012 Accepted: 7 January 2013

Published: 11 January 2013

\section{References}

1. Liu LS: 2010 Chinese guidelines for the management of hypertension. Zhonghua xin xue guan bing za zhi 2011, 39(7):579-615.

2. Bao W, Threefoot SA, Srinivasan SR, Berenson GS: Essential hypertension predicted by tracking of elevated blood pressure from childhood to adulthood: the bogalusa heart study. Am J Hypertens 1995, 8(7):657-665.

3. Cook NR, Gillman MW, Rosner BA, Taylor JO, Hennekens CH: Prediction of young adult blood pressure from childhood blood pressure, height, and weight. J Clin Epidemiol 1997, 50(5):571-579.

4. Mahoney LT, Clarke WR, Burns TL, Lauer RM: Childhood predictors of high blood pressure. Am J Hypertens 1991, 4(11):608S-610S.

5. Daniels SR, Pratt CA, Hayman LL: Reduction of risk for cardiovascular disease in children and adolescents. Circulation 2011, 124(15):1673-1686.

6. Juonala M, Magnussen CG, Berenson GS, Venn A, Burns TL, Sabin MA, Srinivasan SR, Daniels SR, Davis PH, Chen W, et al: Childhood adiposity, adult adiposity, and cardiovascular risk factors. N Engl J Med 2011, 365(20):1876-1885

7. Tu W, Eckert GJ, DiMeglio LA, Yu Z, Jung J, Pratt JH: Intensified effect of adiposity on blood pressure in overweight and obese children. Hypertension 2011, 58(5):818-824

8. Sehested TS, Hansen TW, Olsen MH, Abildstrom SZ, Rasmussen S, Ibsen H, Torp-Pedersen C, Madsbad S, Jeppesen J: Measures of overweight and obesity and risk of cardiovascular disease: a population-based study. European journal of cardiovascular prevention and rehabilitation: official journal of the European Society of Cardiology, Working Groups on Epidemiology \& Prevention and Cardiac Rehabilitation and Exercise Physiology 2010, 17(4):486-490.

9. Sharma AM, Chetty VT: Obesity, hypertension and insulin resistance. Acta Diabetol 2005, 42(Suppl 1):S3-S8.

10. Chen CM: Overview of obesity in mainland China. Obesity reviews: an official journal of the International Association for the Study of Obesity 2008, 9(Suppl 1):14-21 
11. Ji CY: Report on childhood obesity in China (1)-body mass index reference for screening overweight and obesity in Chinese school-age children. Biomedical and environmental sciences: BES 2005, 18(6):390-400.

12. Ji CY, Yt Sung R, Ma GS, Ma J, He ZH, Chen TJ: Waist circumference distribution of Chinese school-age children and adolescents. Biomedical and environmental sciences: BES 2010, 23(1):12-20.

13. Reinehr T, de Sousa G, Toschke AM, Andler W: Long-term follow-up of cardiovascular disease risk factors in children after an obesity intervention. Am J Clin Nutr 2006, 84(3):490-496.

14. Mi JWT, Meng LH, Zhu GJ, Han SM, Zhong Y, Liu GS, Wang YP, Xiong F, Shi $J P$, Yan WL, Zhou PM: Development of blood pressure reference standards for Chinese children and adolescents. Chin J Evid Based Pediatr 2010, 5(1):11.

15. Whitaker RC, Wright JA, Pepe MS, Seidel KD, Dietz WH: Predicting obesity in young adulthood from childhood and parental obesity. N Eng/ J Med 1997, 337(13):869-873.

16. The fourth report on the diagnosis, evaluation, and treatment of high blood pressure in children and adolescents. Pediatrics 2004, 114(2 Suppl 4th Report):555-576.

17. Hypertension control. Report of a WHO expert committee. World Health Organ Tech Rep Ser 1996, 862:1-83.

18. Pall D, Katona E, Fulesdi B, Zrinyi M, Zatik J, Bereczki D, Polgar P, Kakuk G: Blood pressure distribution in a Hungarian adolescent population: comparison with normal values in the USA.J Hypertens 2003, 21(1):41-47.

19. Sun $M, Z$ hou $H Y$, Deng $H Z$ : Study of the risk factors of blood pressure in children. Hunan yi ke da $x$ ue $x u e ~ b a o=$ Hunan yike daxue $x$ uebao = Bulletin of Hunan Medical University 2000, 25(3):238-240.

20. Zhang CX, Shi JD, Huang HY, Feng LM, Ma J: Nutritional status and its relationship with blood pressure among children and adolescents in South China. Eur J Pediatr 2012, 171(7):1073-1079.

21. Hou DQ, Cheng H, Wang TY, Wang L, Zhao D, Zhang MM, Mi J: Study on the relationship of blood pressure with BMI,FMP and waist circumference among children and adolescents in Beijing. Chinese Journal of Practical Pediatrics 2010, 25(7):4.

22. Siani A, Cappuccio FP, Barba G, Trevisan M, Farinaro E, Lacone R, Russo O, Russo P, Mancini M, Strazzullo P: The relationship of waist circumference to blood pressure: the olivetti heart study. Am J Hypertens 2002 15(9):780-786

doi:10.1186/1471-2458-13-24

Cite this article as: Lu et al.: Prevalence of hypertension in overweight and obese children from a large school-based population in Shanghai, China. BMC Public Health 2013 13:24.

\section{Submit your next manuscript to BioMed Central and take full advantage of:}

- Convenient online submission

- Thorough peer review

- No space constraints or color figure charges

- Immediate publication on acceptance

- Inclusion in PubMed, CAS, Scopus and Google Scholar

- Research which is freely available for redistribution 